\title{
Optimised NSAIDs-loaded Biocompatible Nanoparticles
}

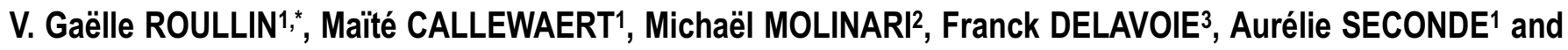 Marie-Christine ANDRY1}

In this formulation study, biocompatible non steroidal anti-inflammatory (NSAIDs)-loaded nanoparticles were designed as models to be further integrated in a prosthesis surface functionalization. A modified spontaneous emulsion-solvent diffusion methodology was used to produce drug-loaded PLGA nanoparticles without any purification or solvent evaporation requirements. Formulation parameters, such as lactide/glycolide ratio, polymer concentration, solvent/non solvent ratio and non solvent phase, as well as the non ionic tensioactive P188 co-precipitation composition were systematically explored. The optimized formulation (mean size: $145 \mathrm{~nm}$, surface charge: $-\mathbf{1 3} \mathrm{mV}$ ) was employed to encapsulate various amounts of NSAIDs in a simple and scalable manner. The drug release was characterized in vitro by a complete release for 48 h. These results encourage upcoming preliminary steps for in vivo experiments of prosthesis surface functionalization.

Keywords: Drug delivery systems (DDS); Biocompatible; Emulsion-solvent diffusion method; PLGA; Glycofurol; Non steroidal anti-inflammatory drugs (NSAIDs)

Citation: V. Gaëlle ROULLIN, Maïté CALLEWAERT, Michaël MOLINARI, Franck DELAVOIE, Aurélie SECOND and Marie-Christine ANDRY “Optimised NSAIDS-loaded biocompatible nanoparticles”, Nano-Micro Lett. 2, 247-255 (2010). doi:10.3786/nml.v2i4.p247-255

The application of polymer-based nanoparticles to exploit biocompatible and biodegradable polymers offers tremendous perspectives as therapeutic vehicles for numerous drugs discarded on the basis of their adverse effects. Indeed, their unique physicochemical properties allow improving drug delivery efficiency to the affected area, and sustaining pharmacological effects [1]. Among those drugs, non steroidal anti-inflammatory drugs (NSAIDs) are especially relevant to such a strategy considering the variety of inflammation pathologies. In particular, it is one of the naturally-occurring processes in joint or bone surgery, when a prosthesis is cemented into the operation site. Perioperative NSAIDs may then be prescribed to reduce the pain in both the short- and long-term surgical outcomes [2] or even to reduce the risk of postoperative ectopic bone formation $[3,4]$. Nevertheless, the oral adminis- tration of NSAIDs is correlated with severe adverse gastrointestinal complications and excessive wound bleedings $[5,6]$. Nanotechnology may confer capability for prolonged and effective delivery of drug as a part of prosthesis surface functionalization.

Such nanoobjects, facing a complex and sensitive biological system as the human body, should meet manifold and extremely challenging requirements, such as biocompatibility, biodegradability, and atoxicity [7]. Furthermore, the production process should be simple, near-net-shape, sterile and easily scalable for cost-effective industrial production.

Following these aforementioned requirements, we aimed at designing NSAIDs-loaded biodegradable nanoparticles to be further coated onto prosthesis surface, in order to allow the local and controlled release of the selected NSAIDs. Poly(lactitde-co-

${ }^{1}$ Institut de Chimie Moléculaire de Reims, CNRS UMR 6229, UFR Pharmacie Reims, 51 rue Cognacq-Jay, 51100 Reims, France

${ }^{2}$ Laboratoire de Microscopies et d'Etudes des Nanostructures, UFR des Sciences, Université de Reims Champagne-Ardenne, 21 rue Clément Ader, 51685 Reims Cedex 2, France

${ }^{3}$ Laboratoire de Microscopie Electronique Analytique, INSERM UMRS 926, 21 rue Clément Ader, 51685 Reims Cedex 2, France

*Corresponding author. E-mail: gaelle.roullin@univ-reims.fr 
glycolide) (PLGA) has been extensively studied for drug delivery applications as nanoparticle matrix material because of its recognized biocompatibility and biodegradability [1]. Various methods have been designed to prepare PLGA nanospheres [1, 7]. Among them, the Spontaneous Emulsification-Solvent Diffusion (SESD) method is a choice methodology since it is simple, low-energetic and reproducible [8]. Nevertheless, it generally implicates at the best class- 3 residual solvents (such as acetone), which involves an additional purification and quantification step. In this preliminary formulation study, we aimed at optimizing this single-step, ready-to-use methodology with the restrained use of completely pharmaceutically-accepted components. A bottom-up, step-by-step strategy was applied to produce NSAIDs-loaded poly-(lactide-co-glycolide) (PLGA) nano- particles, with desirable NSAIDs payloads and release profile. In this respect, the process feasibility and pitfalls were explored through the systematic assessment of formulation parameters and physicochemical characterization.

\section{Materials and methods}

\section{Materials}

Poly(lactide-co-glycolide) (PLGA, 50:50, 65:35, 85:15 lactide / glycolide, molecular weights of 40-75,000, 40-75,000 and 50-75,000 $\mathrm{Da}$, respectively, as provided by the manufacturer) were purchased from Sigma Aldrich. Poloxamer 188 (P188, i.e. Pluronic ${ }^{\circledR} F-68$, polyethyleneglycol-co-polypropyleneglycol-copolyethyleneglycol), the water-miscible solvent glycofurol [9] (i.e. tetraglycol or tetrahydrofurfurylalcohol polyethylene glycol ether) and indomethacin (minimum 99\% TLC) were also obtained from Sigma. Water was exclusively water for injections (Aqua B. Braun, Braun, Germany). Ibuprofen (Fagron, France) was of European Pharmacopeia grade.

\section{Preparation of PLGA nanoparticles}

PLGA nanoparticles were prepared using a modified spontaneous emulsification-solvent diffusion method derived from Niwa et al. [8]. Briefly, the specified mass of PLGA was dissolved in $5 \mathrm{~mL}$ glycofurol, in the presence of various amounts of NSAIDs. This organic phase was poured at a constant flow rate of $2 \mathrm{ml} \cdot \mathrm{min}^{-1}$ to a hydrophilic phase (usually $75 \mathrm{ml}$ ) containing $0.001 \% \mathrm{w} / \mathrm{v}$ poloxamer P188, magnetically stirred at $400 \mathrm{rpm}$. Nanoparticles were spontaneously formed upon solvent phase diffusing into the hydrophilic phase, resulting in a slight blue shade (Tyndall effect). For surface modification, various amounts of poloxamer 188 were dissolved into the organic phase at the same time with the polymer and the drug, adopted from the methodology developed by Csaba et al. [10]. The rest of the experiments were conducted in a strictly similar way.

\section{Nanoparticle size and Zeta potential}

Dynamic light scattering (DLS) was used for the measurement of average hydrodynamic diameters and polydispersity indexes (PdI) (Malvern Zetasizer Nano-ZS, Malvern Instruments, UK). Malvern DTS v4.20 software was used to obtain average hydrodynamic diameters and polydispersity indexes (PdI) with values between 0 and 1 (a value of 0 means that size variation is absent, whereas a PdI value of 1 indicates large variations in particle size distribution). Each sample was analyzed in triplicate at $20^{\circ} \mathrm{C}$ at a scattering angle of $173^{\circ}$.

Zeta-potential data were collected through electrophoretic light scattering at $25^{\circ} \mathrm{C}, 150 \mathrm{~V}$, in triplicate for each sample (Malvern Zetasizer Nano-ZS, Malvern Instruments, UK). The instrument performance was checked with a Malvern-68 mV standard before each analysis cycle.

\section{Morphological studies}

The shape and surface morphology of the PLGA nanoparticles were investigated by transmission electron microscopy (TEM, CM30, Philips, Limeil-Brevannes, France) at an accelerating voltage of $250 \mathrm{kV}$. In prior analysis, samples were negatively stained with $2 \%(\mathrm{w} / \mathrm{v})$ uranyl acetate for $1 \mathrm{~min}$. They were also examined by atomic force microscopy (AFM, Nanoscope III, Veeco, Dourdan, France) in tapping mode (scan rate: $1 \mathrm{~Hz}$ ) using a silicon nitride tip with a constant spring of 46 $\mathrm{N} \cdot \mathrm{m}^{-1}$ and a resonant frequency around $300 \mathrm{kHz}$. Samples were prepared by placing a drop of PLGA nanoparticle suspension on a freshly cleaved mica sheet and allowed to dry in the air. Experiments were performed at room temperature. To obtain Fig. 1(c), after isolating a single nanoparticle, the AFM tip was used to apply a force in the middle of the nanoparticle and then, an image was performed in tapping mode to lower the interaction between the tip and the nanoparticle. 

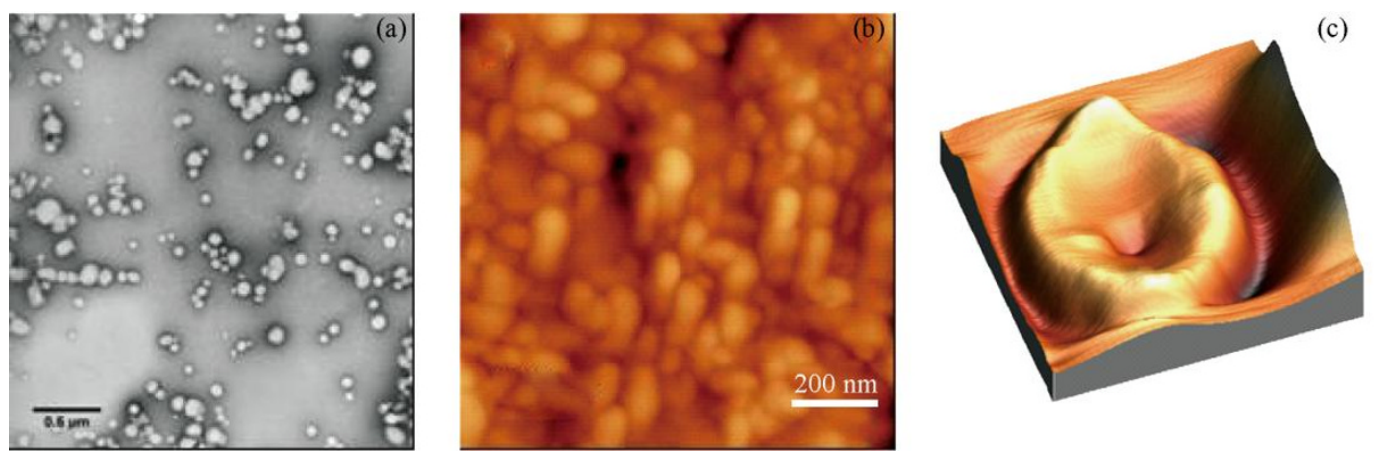

FIG. 1. (a) TEM photographs of nanoparticles prepared from PLGA 50:50, [PLGA] $=1.2 \% \mathrm{w} / \mathrm{v}, \mathrm{S} / \mathrm{NS}=6.6 \% \mathrm{v} / \mathrm{v}$. Bar: $500 \mathrm{~nm}$; (b) AFM surface topography of the same sample obtained in tapping mode $(\mathrm{z}$-scale $=300 \mathrm{~nm})$; (c) 3D-AFM image of a single nanoparticle obtained after applying a force in the middle of the nanoparticle.

\section{NSAIDs quantification}

For drug loading measurement, NSAIDs-loaded nanoparticle suspensions were centrifuged at $23,000 \mathrm{rpm}$ and the suspension supernatants were quantified by HPLC. HPLC analyses were carried out with a C18 column $(250 \times 4.6 \mathrm{~mm} \times 5$ $\mu \mathrm{m})$ mounted on a Dionex system composed of a P680 HPLC Pump, an ASI-100 Automated Sample Injector and a UVD340U detector. For ibuprofen, the mobile phase consisted of a mixture of acetonitrile/water 60/40 containing 1\% o-phosphoric acid. The flow rate was $0.5 \mathrm{ml} / \mathrm{min}$ and peaks were detected as $222 \mathrm{~nm}$. For indomethacin, the mobile phase consisted of a mixture methanol/water $70 / 30$ containing $0.1 \%$ acetic acid. The flow rate was $1 \mathrm{ml} / \mathrm{min}$ and peaks were detected as $225 \mathrm{~nm}$. Chromatograms were evaluated from areas under the curve (AUC) for peaks with a retention time of $5.15 \mathrm{~min}$ for ibuprofen and $18 \mathrm{~min}$ for indomethacin. Calibration curves were performed before and after each quantification cycle, for ibuprofen ranging from 20 to $20,000 \mathrm{ng} / \mathrm{ml}$, for indomethacin from 100 to $10,000 \mathrm{ng} / \mathrm{ml}$.

Entrapment efficiency (EE, \%) of NSAIDs and drug loading efficiency (DLE, \% w/w) were calculated by Equations (1) and (2), respectively:

$$
\begin{aligned}
& E E=\frac{\text { mass of NSAIDs in particles }}{\text { mass of NSAIDs used in formulation }} \times 100 \\
& D L E=\frac{\text { mass of NSAIDs in particles }}{\text { mass of re cov ered particles }} \times 100
\end{aligned}
$$

For the drug release study, $5 \mathrm{ml}$ aliquots of freshly prepared ibuprofen-loaded nanoparticle suspension were placed in a dialysis bag (Spectrum, Spectra/Por ${ }^{\circledR 6}, \mathrm{MWCO}=25,000 \mathrm{Da}$ ) and poured into 11 phosphate buffer $(\mathrm{pH}=7.4)$ in an USP Dissolutest system at $37^{\circ} \mathrm{C}$. The experiment was performed under sink condition to avoid interference from ibuprofen solubility in the release profile in vitro. At regular intervals, $1 \mathrm{ml}$ of release medium was withdrawn and replaced by fresh medium to maintain the final volume constant. The concentration of released drug was measured by HPLC as described previously. Values were reported as the mean $\pm \mathrm{sd}$ of four replicates.

\section{Results and discussion}

Prior examining and discussing the outcomes of various factors on the nanoparticle formation, it is important to state that spherical, individualized nanoparticles were obtained in a wide range of process conditions with fabrication yields over $90 \%$ (Fig. 1). TEM photographs showed a well-defined spherical particle shape, without noticeable aggregation (Fig. 1(a)). AFM images showed smooth nanoparticle surface (Fig. 1(b)). AFM also enabled us to precise the core-shell structure of the nanoparticles, as demonstrated by the possibility for the AFM tip to depress them upon contact (Fig. 1(c)). Nevertheless, it was not possible, with the use of present equipment, to quantify these phenomena.

\section{Influence of process parameters on the nanoparticle characteristics}

For efficient drug delivery, it is necessary that nanoparticle mean sizes and population polydispersity indexes should be kept as low as possible. In this work, hydrodynamic diameters were considered suitable for the potential therapeutic applications if mean values were less than or equal to $200 \mathrm{~nm}$ and polydispersity indexes (as calculated by the Malvern DTS v 4.20 software) less than 0.12 , which indicates monodisperse size distributions. Indeed mean sizes should be kept under $200 \mathrm{~nm}$ to facilitate surface prosthesis integration, for example, in a hydroxyapatite trabecular meshwork coating a titane surface prosthesis [11]. Furthermore, a constant regular drug release is 
desirable for the considered therapeutic application, which can be reached through monodisperse size populations [12]. Preliminary studies on nanoparticle formation revealed that, to reach this goal, the critical factors during processing were lactide-to-glycolide ratio and polymer concentration. Other factors with great impacts on the final results were also examined, such as solvent/non-solvent volume ratio and tensioactive co-precipitation. Herein, solvent and non solvent refer to the ability to solubilize the polymer, solvent being glycofurol and non solvent being water, more or less enriched with tensioactive molecules.

\section{$\mathrm{L} / \mathrm{G}$ ratio}

The influence of the lactide-to-glycolide $(\mathrm{L} / \mathrm{G})$ ratio on particle size is summarized in Table 1, for similar molecular weights and a fixed polymer concentration. A clear trend of increasing particle sizes (as well as polydispersity indexes, PdIs) with increasing the $\mathrm{L} / \mathrm{G}$ ratio was observed. This phenomenon was previously reported with many types of polyester dissolved in a large variety of solvents, in the solvent diffusion process [13, 14]. In fact, an increase of the $\mathrm{L} / \mathrm{G}$ induces an increase of polymer hydrophobicity due to a higher lactide percentage. This would, in turn, result in increased polymer-polymer interactions, and consequently larger particles. Finally, a higher proportion of lactide units leads to a larger relative amount of crystalline micro-domains in its solid state [15]. When the polymer precipitates during desolvation, such lactide domains would present more difficulties to fold and re-arrange in dense spherical features, leading globally to bigger particles. For these reasons, PLGA 50:50 was selected as the most suitable polymer in this study.

\section{Polymer concentration}

As already described, polymer concentration in the solvent phase, and hence viscosity, greatly affects the observed sizes [16-18]. The mechanism of particle formation during spontaneous emulsification occurs according to "diffusionstranding" process, also known as the Marangoni effect [18-20]. In this respect, the polymer concentration was varied in order to alter the inner solvent phase viscosity and, consequently the solvent diffusion rate into the non-solvent phase [17]. Contrary to what was generally observed [17-21], a linear response between polymer concentration and hydrodynamic diameters was not observed in this study (Fig. 2(a)). On the opposite, there seemed to be a critical PLGA concentration (with our protocol $2 \% \mathrm{w} / \mathrm{v}$ ) above which the hydrodynamic diameters and PdIs were characterized by erratic values. This phenomenon was already observed in the case of nanoparticles produced by spraying PLGA-glycofurol solution into water [22]. It was as if the solvent molecules were being able to diffuse normally into the non-solvent phase until a critical polymer concentration was reached, which blocked the small solvent molecules into the folded polymer chains. This could demonstrate that the diffusion process is hampered by the affinity between solvent molecules and polymer chains, i.e. the balance between forces involved into polymer solvation and the ones involved into solvent diffusion, as stated by Choi et al. [23]. Further investigations were therefore conducted with a $1.5 \%$ PLGA concentration.

\section{Solvent/Non-solvent phases (S/NS) ratio}

Table 1 Effect of some formulation parameters on sizes and zeta potentials of raw blank nanoparticles

\begin{tabular}{|c|c|c|c|c|}
\hline $\begin{array}{c}\text { PLGA } \\
\text { L/G ratio }\end{array}$ & NS phase composition & $\begin{array}{c}\text { Mean diameters } \\
\mathrm{nm} \\
( \pm \mathrm{sd}) \\
\end{array}$ & $\begin{array}{c}\text { PdI } \\
( \pm \mathrm{sd}) \\
\end{array}$ & $\begin{array}{c}\text { Zeta potentials } \\
\mathrm{mV} \\
( \pm \mathrm{sd}) \\
\end{array}$ \\
\hline $85: 15$ & Water : P18 $8^{\mathrm{a}}$ & $\begin{array}{c}239 \\
(108)^{\mathrm{b}}\end{array}$ & $\begin{array}{c}0.622 \\
(0.28)^{\mathrm{b}}\end{array}$ & $\begin{array}{l}-18.2 \\
(0.6)\end{array}$ \\
\hline $65: 35$ & Water : P188 & $\begin{array}{c}234 \\
(33)^{\mathrm{b}}\end{array}$ & $\begin{array}{c}0.188 \\
(0.07)^{\mathrm{b}}\end{array}$ & $\begin{array}{l}-20.4 \\
(0.5)\end{array}$ \\
\hline $50: 50$ & Water : P188 & $\begin{array}{l}149 \\
(8)\end{array}$ & $\begin{array}{l}0.107 \\
(0.01)\end{array}$ & $\begin{array}{l}-17.3 \\
(0.5)\end{array}$ \\
\hline & Water & $\begin{array}{l}164 \\
(17) \\
\end{array}$ & $\begin{array}{l}0.128 \\
(0.05) \\
\end{array}$ & $\begin{array}{l}-25.9 \\
(2.5) \\
\end{array}$ \\
\hline
\end{tabular}

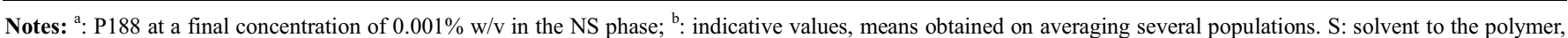
i.e. glycofurol ; NS: non solvent to the polymer, i.e. water. 

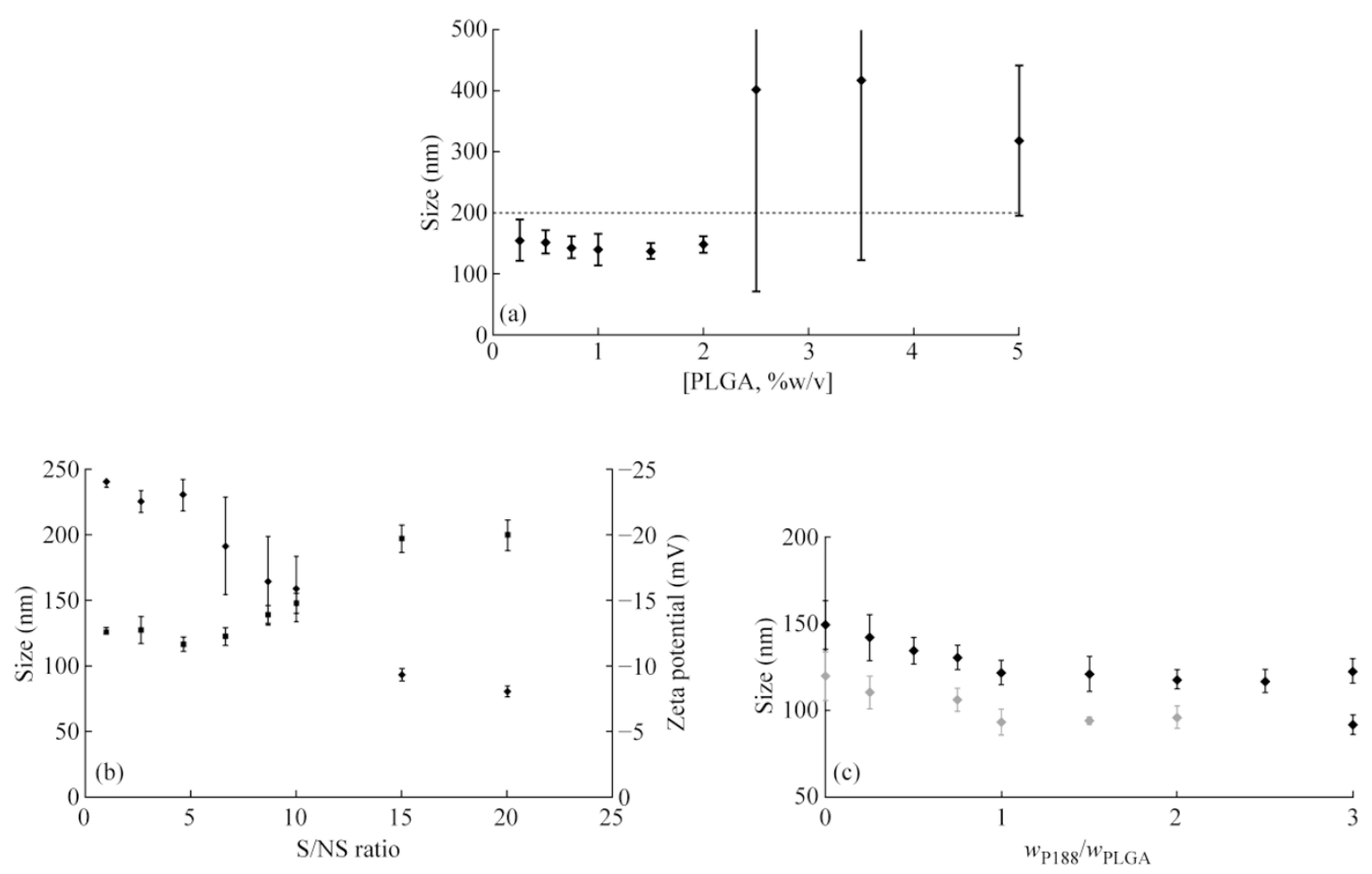

FIG. 2. Evolution of nanoparticle sizes (hydrodynamic diameters, $\mathrm{nm}$ ) as a function of: (a) polymer concentrations (\% w/v) at constant $\mathrm{S} / \mathrm{NS}$ ratio (6.6\% v/v); (b) as a function of $\mathrm{S} / \mathrm{NS}($ glycofurol/water) phase ratio $(\%, \mathrm{v} / \mathrm{v})$ at constant polymer concentration $(1.5 \% \mathrm{w} / \mathrm{v})$ and corresponding zeta potentials (orange points, $\mathrm{mV})$; (c) as a function of P188 / PLGA ratio (w/w) at constant polymer concentration (1.5\% w/v), before (black) and after dialysis (blue).

Results are given as means $\pm \mathrm{sd}, \mathrm{n}=3$ to 9 .

The effect of varying the solvent (glycofurol)-to-non solvent (water) volume ratio (S/NS) during polymer precipitation was investigated, from 1 to $20 \%$ (v/v) (Fig. 2(b)). Overall, for a fixed 1.5\% PLGA concentration, sizes decreased with decreasing the S/NS ratio, until a minimum was reached around $7 \%$. In our system, phase transfer was not instantaneous since glycofurol was more viscous than water (8-18 vs $1 \mathrm{~m} \cdot \mathrm{Pa} \cdot \mathrm{s}$ at $20^{\circ} \mathrm{C}$ [9]), resulting in a transient solvent concentration gradient into the non-solvent phase. Even if sink conditions were globally assumed, increasing the glycofurol/water ratio led to larger particles. This could be due to a locally solvent-saturated system at the interface, slowing down glycofurol diffusion [23] and therefore tending to favour nanodroplet collapses. This was partially visible through the evolution of zeta-potentials (Fig. 2(b)), which were far more negative for lower ratios than higher ones. This testifies for the presence of glycofurol molecules contributing to the double solvation layer when desolvation is not complete. Nevertheless, for a large scale of volume ratios, particles remained in the range of $200 \mathrm{~nm}$ or less. The presence of a plateau in the size evolution (for glycofurol/water ratio smaller than $7 \% \mathrm{v} / \mathrm{v}$ ) reveals the existence of a maximal surface curvature, which could not be increased whatever the ratio.

\section{Influence of tensioactive-polymer co-precipita- tion}

The development of stealth nanoparticles as drug carriers, which could avoid or at least reduce the uptake by phagocytes and therefore an immunogenic response, has been thoroughly examined in the last decade $[24,25]$. It is now well established that the adsorption of blood proteins (leading to surface opsonisation) on hydrophilic surfaces is greatly delayed compared to hydrophobic ones [25]. Surface adsorption or polymer grafting are general methods of choice to turn an hydrophobic surface (such as PLGA) into a more hydrophilic one, using either long hydrophilic polymer chains (like polysaccharides or PEG) or non-ionic surfactants (for example PVAL, poloxamers and poloxamines). In this prospect, the co-precipitation approach developed by Alonso and co-workers is particularly interesting. They initiated a technique in which either poloxamines (Tetronic ${ }^{\circledR} 904$ and 908 ) or poloxamers (Pluronic ${ }^{\circledR}$ F68 and L121) were co-dissolved in the solvent phase and nanoprecipitated into an aqueous phase [26]. During precipitation, the tensioactive molecules placed at the solvent non solvent interface were trapped by physical entanglements with polymer chains. In this study, we applied this approach to 
obtain stealth nanoparticles, for which results are shown in Fig. 2(c).

The far less negative zeta-potentials observed on these particles $([-20 ;-30] \mathrm{mV})$, after the elimination of the tensioactive excess by dialysis, as compared to bare nanoparticles ([-50; -55] $\mathrm{mV}$ ), is due to the presence of entrapped P188 molecules masking the terminal outer carboxylic charges on the PLGA nanoparticle surface. Increasing P188 concentration was performed in order to try to maximise the surface density of the stabilizing agent. Indeed, it has been previously reported that it would reduce the uptake of particles by the RES [24], without affecting the drug entrapment efficiency [10].

Sizes diminished with increasing P188 ratio in the glycofurol phase (Fig. 2(c)). Indeed, the more P188 molecules at the nanoparticle surface, the higher the hydrodynamic radius would appear, according to the Stockes-Einstein equation. The presence of the P188 tensioactive molecules, displaying a higher affinity for water than for glycofurol, must contribute to stabilize the spontaneous oil-in-water emulsion, leading to smaller micelle-like structures. Then upon polymer desolvation, the resulting solid particles present a smaller hydrodynamic diameter. This can happen until the limit is reached (Fig. 2(c)), limit which is controlled by: 1-the presence of polymer chains inside the nanodroplets; 2-the maximal surface curvature observed for this system.

\section{NSAIDs encapsulation}

NSAIDs-loaded nanoparticles were prepared from the optimized formulation, with a PLGA $_{50: 50}: \mathrm{P} 188$ 1:1 ratio and a S/NS volume ratio of $6.6 \%$. In the absence of P188 in the S

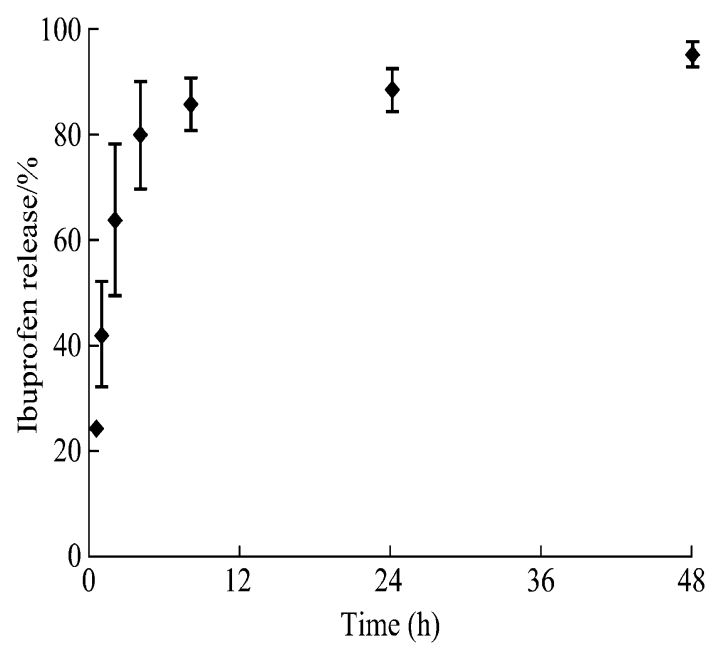

FIG. 3. Ibuprofen release kinetics from 27\%-ibuprofen loaded PLGA nano-

particles. Mean values $\pm \mathrm{sd}, \mathrm{n}=4$.

(glycofurol) phase, total nanoparticle flocculation occurred between 0 and $24 \mathrm{~h}$ post-synthesis. In the presence of $\mathrm{P} 188$, the nanoparticle recovery yields varied with the drug concentration in the $\mathrm{S}$ phase as well as the observed sizes, while zeta-potentials remained globally unchanged (Table 2). Concerning the encapsulation efficiencies (EE) and drug loading efficiencies (DLE) for ibuprofen, the best compromise was obtained for a $3 \%$ $\mathrm{w} / \mathrm{v}$ drug concentration in the solvent phase. For indomethacin, best results were obtained with $0.2 \%$ introduced in the $\mathrm{S}$ phase, leading to mean entrapment efficiency of $64 \%$ after dialysis and a similar DLE value with ibuprofen. Those data are comparable to those obtained by others [27-30].

Concerning the ibuprofen-loaded nanoparticles, the cumulative drug release occurred over a 2-day period and displayed a biphasic pattern. First, an initial burst up to $80 \%$ was

Table 2 Characterization of NSAIDs encapsulation with the optimized formulation (means of $n=3$, standard deviations between brackets)

\begin{tabular}{|c|c|c|c|c|c|c|c|}
\hline & \multicolumn{2}{|c|}{ Drug quantity } & \multirow{2}{*}{$\begin{array}{c}\text { Size } \\
\mathrm{nm} \\
( \pm \mathrm{sd})\end{array}$} & \multirow[b]{2}{*}{ PdI } & \multirow{2}{*}{$\begin{array}{c}\square \text { zetappotentials } \mathrm{mV} \\
( \pm \mathrm{sd})\end{array}$} & \multirow{2}{*}{$\begin{array}{c}\mathrm{EE}^{\mathrm{a}} \\
\% \\
( \pm \mathrm{sd})\end{array}$} & \multirow{2}{*}{$\begin{array}{c}\text { DLE }^{\mathrm{b}} \\
\%\end{array}$} \\
\hline & $\begin{array}{c}\text { in S phase, } \\
\% \mathrm{w} / \mathrm{v} \\
\end{array}$ & $\begin{array}{c}\text { Mass, } \\
\mathrm{mg}\end{array}$ & & & & & \\
\hline \multirow{5}{*}{ ibuprofen } & 0 & 0 & $\begin{array}{c}144 \\
(0.2)\end{array}$ & $\begin{array}{l}0.107 \\
(0.01)\end{array}$ & $\begin{array}{l}-12.8 \\
(0.2)\end{array}$ & - & - \\
\hline & 0.44 & 22 & $\begin{array}{l}157 \\
(0.5)\end{array}$ & $\begin{array}{c}0.08 \\
(0.01)\end{array}$ & $\begin{array}{l}-10.5 \\
(0.1)\end{array}$ & $\begin{array}{l}30 \\
(8)\end{array}$ & 12 \\
\hline & 3 & 150 & $\begin{array}{l}148 \\
(3)\end{array}$ & $\begin{array}{c}0.09 \\
(0.02)\end{array}$ & $\begin{array}{l}-11.8 \\
(0.5)\end{array}$ & $\begin{array}{l}27 \\
(6)\end{array}$ & 45 \\
\hline & 4 & 200 & $\begin{array}{l}194 \\
(3)\end{array}$ & $\begin{array}{c}0.13 \\
(0.04)\end{array}$ & $\begin{array}{l}-12.1 \\
(0.5)\end{array}$ & $\begin{array}{l}24 \\
(6)\end{array}$ & 49 \\
\hline & 8 & 400 & $\begin{array}{l}183 \\
(4)\end{array}$ & $\begin{array}{c}0.12 \\
(0.02)\end{array}$ & $\begin{array}{l}-12.9 \\
(1.1)\end{array}$ & $\begin{array}{l}12 \\
(1)\end{array}$ & 49 \\
\hline \multirow{2}{*}{ indomethacin } & 0.06 & 3 & $\begin{array}{l}133 \\
(1)\end{array}$ & $\begin{array}{c}0.07 \\
(0.01)\end{array}$ & $\begin{array}{l}-20.6 \\
(0.5)\end{array}$ & $\begin{array}{c}38 \\
(0.5)\end{array}$ & 1.6 \\
\hline & 0.2 & 10 & $\begin{array}{l}129 \\
(1)\end{array}$ & $\begin{array}{c}0.14 \\
(0.01)\end{array}$ & $\begin{array}{l}-35.6 \\
(4.3)\end{array}$ & $\begin{array}{c}64 \\
(15)\end{array}$ & 13 \\
\hline
\end{tabular}

${ }^{\mathrm{a}}$ EE: encapsulation efficiency; ${ }^{\mathrm{b}}$ DLE: drug loading efficiency. 


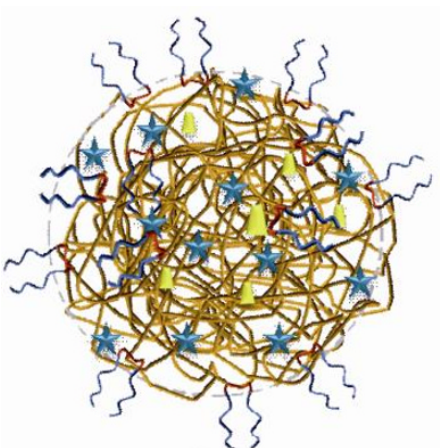

(a)

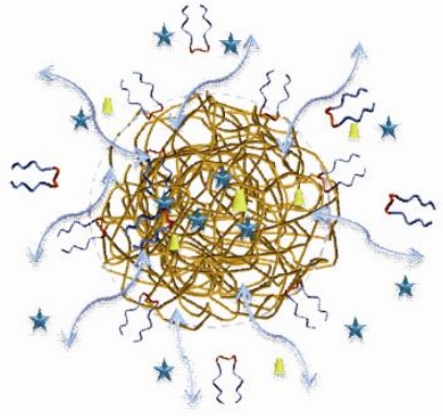

(b)

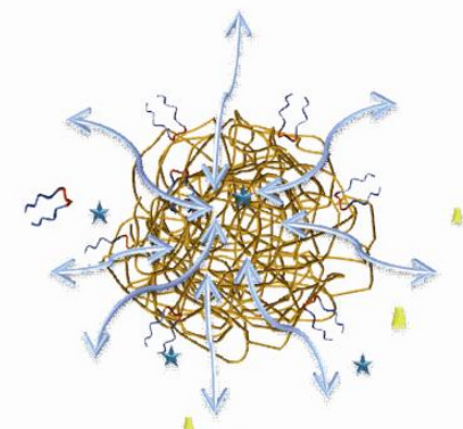

(c)

FIG. 4. Scheme of hypothesized ibuprofen release mechanisms: (a) state of ibuprofen (green stars) and glycofurol (yellow cones) molecules immobilized in PLGA (orange) and P188 (blue/orange) segments at time =0; (b) rapid release of the surface molecules by water flow (blue arrows) in the first minutes; (c) deeper penetration of water molecules and subsequent slower release for times $>4 \mathrm{~h}$.

observed for $4 \mathrm{~h}$ (Fig. 3), corresponding to a large proportion of drug present at or near the nanoparticle surface (Fig. 4(a) and (b)). Indeed, in equivalent experimental conditions, the recovery of the same quantity of free ibuprofen dissolved in a blank NP suspension was completed in $2 \mathrm{~h}$ (data not shown), whereas only about $60 \%$ were released in the case of ibuprofen-loaded nanoparticles (Fig. 3). The rest (about 20\%) must be located near the surface and then be washed out, with the adsorbed / dispersed part, by the water flow penetrating the polymer network (Fig. 4(b)). It was followed by a much slower phase (Fig. 3), due to the entrapment of the drug deeper into the PLGA/P188 matrix (Fig. 4(c)). As a matter of fact, to access the deeper core of the nanoparticle, water molecules have to create pathways and to exchange with solvent (glycofurol) molecules, which takes more time and may account for this slower release phase. The complete release is achieved in $48 \mathrm{~h}$.

Several factors affect the shape of the release pattern [31, 32]. The drug release from PLGA particles may depend on three primary mechanisms, i.e. polymer swelling, drug diffusion through polymer network, and polymer degradation via hydrolysis [32]. For high molecular weight PLA and PLGA systems, the diffusion process is dominant and principally drives the initial burst release mechanisms [33]. The second phase is more generally attributed to uniform degradation and erosion of the particle wall, modulated through the solvent diffusion and subsequent enrichment of the external medium [34]. Surprisingly, the role of the nature and diffusion kinetics of the remaining solvent molecules inside the nanoparticles is scarcely discussed. In the case of nanocapsules, this role cannot be neglected since it constitutes a major component of the diffusional system. Thus, the remaining glycofurol molecules must contribute to plasticize the polymer chains and to solubilize the drug, modulating the NSAID release. Furthermore, this phenomenon would be re-enforced by the presence of PEG segments (from co-precipitated P188 molecules) [33], since the polymer network would be enlarged and facilitate drug diffusion to external medium. Nevertheless, the release pattern observed herein is comparable to what others reported about NSAIDs of the same chemical natures [35-37]. Indeed there seems to be a general finding that PLGA nanoparticles within the 200-nm range display a complete release less than $24 \mathrm{~h}$ when loaded with such chemicals [7]. For prosthesis surface coating, such a release pattern should be adequate since, occurring in a larger period than $24 \mathrm{~h}$, it could advantageously replace an oral daily preventive cure.

\section{Conclusion}

The aim of the study was to design stealth NSAID-loaded polymeric nanoparticles according to a one-step nanoprecipitation process to be further coated onto prosthesis surface for drug controlled release. To our knowledge, the use of parenteral solvent glycofurol to produce PLGA-based drug delivery systems is scarce and mainly concerns with macro- or microscopic systems [38-41]. Glycofurol-based nanoparticles have only been reported once before this work, by spraying PLGA solution into water, with a lesser success in terms of particle production, size and drug loading efficiency [22].

In this work, the influence of various processing variables on particle characteristics was systematically assessed and the optimised formulation parameters were applied to a successful encapsulation of NSAIDs. Compared to previous reported approaches [27,28,30], an appreciable amount of drug was encapsulated and a suitable rate of drug release was achieved, with a complete release in 2 days, which could advantageously replace a daily oral administration. Preliminary in vitro assays on murine macrophages, presently on-going, confirm their non 
toxicity, which opens tracks to further in vivo investigations. In vivo characteristics of this nanosized system should show its effetiveness as a part of prosthesis surface functionalisation, for useful NSAIDs local release.

The authors thank Pr Françoise Chuburu for the revision of this manuscript and her helpful remarks. Experiments were partly realized by Agnieszka Mrall and Cristina Giammichele, financially supported by the European Erasmus program.

\section{Received 12 October 2010; accepted 22 November; published online 3 December.}

\section{References}

1. A. Kumari, S. Kumar Yadav and S. C. Yadav, Colloids Surf. B 75, 1 (2010). doi:10.1016/j.colsurfb.2009.09.001

2. V. Dahl, J. C. Raede, S. Drosdal, O. Wathne and J. Brynildsrud, Acta Anaesthesiol. Scand. 39, 323 (1995). doi:10.1111/j.1399-6576.1995.tb04070.x

3. M. Fransen, Control. Clin. Trials 25, 223 (2004). doi:10.1016/j.cct.2003.11.008

4. C. L. Romano, D. Duci, D. Romano, M. Mazza and E. Meani, The Journal of Arthroplasty 19, 14 ( 2004). doi:10.1016/S0883-5403(03)00279-1

5. M. Fransen, C. Anderson, J. Douglas, S. MacMahon, B. Neal, R. Norton, M. Woodward, I. D. Cameron, R. Crawford, S. K. Lo, G. Tregonning and M. Windolf, BMJ. 333, 519 ( 2006). doi:10.1136/bmj.38925.471146.4F

6. J. P. Cella, E. A. Salvati and T. P. Sculco, The Journal of Arthroplasty 3, 229 (1988).

7. C. E. Mora-Huertas, H. Fessi and A. Elaissari, Int. J. Pharm. 385, 113 (2010). doi:10.1016/j.ijpharm.2009.10. $\underline{018}$

8. T. Niwa, H. Takeuchi, T. Hino, N. Kunou and Y. Kawashima, J. Controlled Release 25, 89 (1993) doi:10. $\underline{1016 / 0168-3659(93) 90097-O}$

9. P. J. Weller, "Handbook of Pharmaceutical Excipients 5th edition”, London: Pharmaceutical Press, 2006.

10. N. Csaba, P. Caamaño, A. Sánchez, F. Dominguez and M. J. Alonso, Biomacromolecules 6, 271 (2005). doi:10. $\underline{1021 / \mathrm{bm} 049577 \mathrm{p}}$

11. O. Albayrak, O. El-Atwani and S. Altintas, Surf. Coat. Technol. 202, 2482 (2008). doi:10.1016/j.surfcoat.2007. $\underline{09.031}$

12. K. Y Win and S. S. Feng, Biomaterials 26, 2713 (2005). doi:10.1016/j.biomaterials.2004.07.050
13. H. Gao, Y. N. Wang, Y. G. Fan and J. B. Ma, J. Biomed. Mater. Res. Part A 80, 111 (2007). doi:10.1002/ jbm.a.30861

14. T. Riley, S. Stolnik, C. R. Heald, C. D. Xiong, M. C. Garnett, L. Illum and S. S. Davis, Langmuir 17, 3168 (2001). doi:10.1021/la001226i

15. G. Mittal, D. K. Sahana, V. Bhardwaj and M. N. V. Ravi Kumar, J. Controlled Release 119, 77 (2007). doi:10.1016 /i.jconrel.2007.01.016

16. D. Quintanar-Guerrero, E. Allemann, H. Fessi and E. Doelker, Int. J. Pharm. 188, 155 (1999). doi:10.1016/ S0378-5173(99)00216-1

17. T. Jung, A. Breitenbach and T. Kissell, J. Controlled Release 67, 157 (2000). doi:10.1016/S0168-659(00) $\underline{002017}$

18. J. Cheng, B. A. Teply, I. Sherifi, J. Sung, G. Luther, F. X. 3Langer and O. C. Farokhzad, Biomaterials 28, 869 (2007). doi:10.1016/j.biomaterials.2006.09.047

19. D. Quintanar-Guerrero, E. Allemann and E. Doelker, Colloid Polym. Sci. 275, 640 (1997). doi:10.1007/s00396 0050130

20. N. Anton, J. P. Benoit and P. Saulnier, J. Controlled Release 128, 185 (2008). doi:10.1016/j.jconrel. 2008.02.007

21. A. Aumelas, A. Serrero, A. Durand, E. Dellacherie and M. Leonard, Colloids Surf. B 59, 74 (2007). doi:10.1016/i.col $\underline{\text { surfb.2007.04.021 }}$

22. B. K. Kim, D. Kim, S. H. Cho and S. H. Yuk, J. Microencapsulation 21, 697 (2004). doi:10.1080/0265 $\underline{2040400000520}$

23. S. W. Choi, H. Y. Kwon, W. S. Kim and J. H. Kim, Colloids Surf. A 201, 283 (2002). doi:10.1016/ $\underline{\mathrm{S} 09277757(01) 01042-1}$

24. F. Gu, L. Zhang, B. A. Teply, N. Mann, A. Wang, A. F. Radovic-Moreno, R. Langer and O. C. Farokhzad, Proc. Natl. Acad. Sci. U. S. A. 105, 2586 (2008). doi:10.1073/ pnas.0711714105

25. C. Passirani, G. Barratt, J. P. Devissaguet and D. Labarre, Pharm. Res. 15, 1046 (1998). doi:10.1023/A:1011 $\underline{930127562}$

26. N. Csaba, L. González, A. Sánchez and M. J. Alonso, J. Biomater. Sci. Polym. Ed. 1137 (2004). doi:10.1163/ 1568562041753098

27. B. Jiang, L. Hu, C. Gao and J. Shen, Int. J. Pharm. 304, 220 (2005). doi:10.1016/j.ijpharm.2005.08.008 
28. M. A. Casadei, F. Cerreto, S. Cesa, M. Giannuzzo, M. Feeney, C. Marianecci and P. Paolicelli, Int. J. Pharm. 325, 140 (2006). doi:10.1016/j.ijpharm.2006.06.012

29. M. Feng, P. Li, J. Biomed. Mater. Res. Part A 80, 184 (2007). doi:10.1002/jbm.a.30882

30. A. Sheikh Hasan, M. Socha, A. Lamprecht, F. E. Ghazouani, A. Sapin, M. Hoffman, P. Maincent and N. Ubrich, Int. J. Pharm. 344, 53 (2007). doi:10.1016 /j.ijpharm.2007.05.066

31. F. Alexis, Polym. Int. 54, 36 (2005). doi:10.1002/pi.1697

32. N. Faisant, J. Siepmann and J. P. Benoit, Eur. J. Pharm. Sci. 15, 355 (2002). doi:10.1016/S0928-0987(02)00023-4

33. J. Siepmann and F. Siepmann, Int. J. Pharm. 364, 328 (2008). doi:10.1016/j.ijpharm.2008.09.004

34. B. S. Zolnik, P. E.Leary and D. J. Burgess, J. Controlled Release 112, 293 (2006). doi:10.1016/j.jconrel. $\underline{2006.02 .015}$

35. E. Vega, F. Gamisans, M. L. Garcia, A. Chauvet, F. Lacoulonche and M. A. Egea, J. Pharm. Sci. 97, 5306 (2008). doi:10.1002/jps.21383
36. Y. Javadzadeh, F. Ahadi, S. Davaran, G. Mohammadi, A. Sabzevari and K. Adibkia, Colloids Surf. B 81, 498 (2010). doi:10.1016/j.colsurfb.2010.07.047

37. D. Klose, F. Siepmann, K. Elkharraz and J. Siepmann, Int. J. Pharm. 354, 95 (2008). doi:10.1016/j.ijpharm. $\underline{2007.10 .030}$

38. W. J. Cho, J. H. Kim, S. H. Oh, H. H. Nam, J. M. Kim and J. H. Lee, J. Biomed. Mater. Res., Part A 91, 400 (2009) doi:10.1002/jbm.a.32264

39. R. E. Eliaz and J. Kost, J. Biomed. Mater. Res. 50, 388 (2000).

40. A. Aubert-Pouëssel, M. C. Venier-Julienne, P. Saulnier, M. Sergent and J. P. Benoît, Pharm. Res. 21, 2384 (2004). doi:10.1007/s11095-004-7693-3

41. D. Allhenn and A. Lamprecht, Pharm. Res. (2010). doi:10.1007/s11095-010-0304-6 